\title{
Chronic Subdural Hematoma Presenting with Kernohan-Woltman Notch Phenomenon: A Case Report
}

\author{
Bin Alhassan, E Gamlie, R Hagan and K Agyen Mensah* \\ Department of Surgery, University of Cape Coast, Ghana \\ *Corresponding author: Kwasi Agyen Mensah, Department of Surgery, School of Medical Sciences University of Cape Coast, Ghana
}

Submission: August 07, 2018; Published: September 12, 2018

\begin{abstract}
Kernohan-Woltman Notch Phenomenon (KWNP) is a neurologic picture caused by the compression of the contralateral cerebral peduncle on the free edge of the tentorium resulting from the displacement of the brain tissue at the ipsilateral side of a paradoxically supratentorial lesion leading to mydriasis and hemiparesis or hemiplegia ipsilateral to a supratentorial space occupying mass [1]. This phenomenon is usually reported secondary to brain tumors and severe head injuries [2]. The development of KWNP secondary to chronic subdural hematomas has been rarely reported and there is no reported case from Ghana where this case is being reported from. We report this case of Kernohan-Woltman notch phenomenon in a 50 year old man with a chronic subdural hematoma who was brought to the Emergency Department in a comatose state.
\end{abstract}

Keywords: Kernohan woltman notch; Kernohan's phenomenon; Brain herniation; Brain shift; Chronic subdural hematoma

\section{Introduction}

Kernohan-Woltman notch phenomenon (KWNP) is defined as compression of the cerebral peduncle against the tentorial edge caused by the displacement of the brain tissue at the ipsilateral side of the paradoxically supratentorial-localized lesion such that it may lead to hemiparesis or hemiplegia and mydriasis [1]. This phenomenon is usually reported secondary to brain tumors and severe head injuries [2]. The development of KWNP secondary to acute on chronic subdural hematomas has been rarely reported and there is no reported case from Ghana where this case is being reported from.

There are some reported cases of chronic subdural hematoma with Kernohan's notch phenomenon in literature but to the best of the authors' knowledge there are no reported cases from Ghana and West Africa.

In a review article by Zhang et al. [3] out of thirty-nine cases from a PubMed search thirty six were due to intracranial bleeds the rest of which were as a result of arachnoid cyst, high grade glioma and reabsorption bone syndrome.

\section{Case Report}

A 50-year-old male patient was seen at the Emergency Department of the Cape Coast Teaching Hospital with a history of sudden loss of consciousness. He had been experiencing headaches a couple of weeks prior to this presentation. Two months earlier, the patient was riding his motorcycle when he lost control, accelerated and hit a palm tree with him still sitting on the motorbike. He recollects hitting the head against the palm tree but did not lose consciousness. He was treated and discharged at a local clinic. Three weeks later he started experiencing headaches for which he took over the counter analgesics thinking it was an ordinary headache; only for him to dip in consciousness suddenly on the day of presentation. On examination, his Glasgow coma score (GCS) was $7 / 15$; best motor score of 5 , verbal response of 1 and eye-opening response of 1 and had a left hemiplegia. The left pupil was $4 \mathrm{~mm}$ in diameter and reacted sluggishly to light, but the right pupil was $2 \mathrm{~mm}$ with a sluggish reaction to light.

A non-contrast head computed tomography scan showed a left front-parietal chronic subdural hematoma with marked midline shift as depicted in (Figure $1 \& 2$ ). A clinical diagnosis of KernohanWoltman notch phenomenon was made. Emergency burr hole drainage of the chronic subdural hematoma using a double burr hole technique was done. Patient regained consciousness twelve hours post-operatively but was noticed to have an ipsilateral left hemiparesis and a persistent left eye mydriasis. The patient was discharged home on post-operative day 5 after full recovery with no residual neurological deficits. The patient on follow up visit three (3) weeks after surgery was found to be doing well with no residual neurological deficit. 


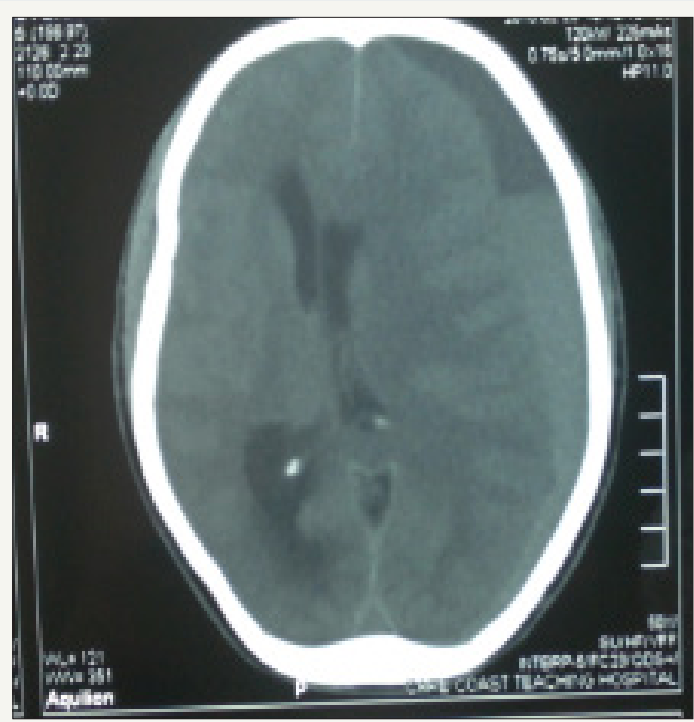

Figure 1: A left frontoparietal chronic subdural hematoma with a sub-acute component showing significant midline shift.

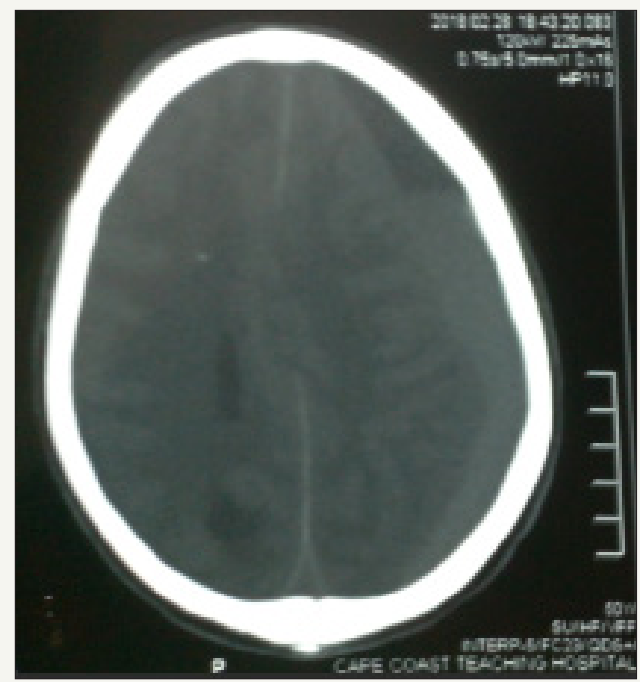

Figure 2: Axial CT scan at a different level with left frontoparietal chronic subdural hematoma showing a midline shift.

\section{Discussion}

Kernohan and Woltman described in 1929 that any supratentorial-localized mass may compress the contralateral cerebral peduncles at the tentorial edge [3] Injury of corticospinal motor neurons in the contralateral cerebral peduncles may also cause the false localizing sign, thus defining a Kernohan-Woltman Notch Phenomenon (KWNP) [4]

Chronic subdural hematomas usually occur among old patients and alcoholics following head trauma even when the trauma is a trivial one due to brain atrophy and stretching of the bridging veins. Patients on anticoagulants are also prone to chronic subdural hematoma. Much less common causes of subdural hematoma include coagulopathies, seizure disorders, cerebrospinal fluid (CSF) shunts and ruptured intracranial aneurysms. The subdural space lies between the inner surface of the dura mater and the outer arachnoid layer. Bridging veins travel from the convexities of the cerebral hemispheres through the subarachnoid space and the subdural space to empty into the superior sagittal sinus. Similar anatomic relationships exist with other dural sinuses. These vessels are usually prone to tearing along their course through the subdural space. They are the source of bleeding in most cases of subdural hematoma. Since the brain floats freely within the cerebrospinal fluid (CSF), it can move within the skull, however, the venous sinuses are fixed. The displacement of the brain that occurs in trauma can tear the veins at the point where they penetrate the dura.

Patients with chronic subdural hematomas tend to have decreased blood flow to the thalamus and basal ganglia as compared to other areas of the brain. Tanaka et al found that a seven percent $(7 \%)$ decrease of cerebral blood flow (CBF) was commonly associated with headache, whereas a thirty five percent (35\%) decrease of CBF was associated with neurologic deficit such as hemiparesis [5]

The diagnosis of KWNP in our patient was made 12 hours post-operative when he had regained consciousness and was noticed to have a residual weakness of the ipsilateral left upper and 
lower limbs and mildly dilated left pupil which reacted sluggishly to light. The tentative diagnosis of a Kernohan- Woltman notch phenomenon was confirmed after surgery when a more detailed neurological examination was done after stabilizing the patient. Per the knowledge of the writers, this is the first reported case of chronic subdural hematoma with Kernohan's Woltman Notch Phenomenon in Ghana and West Africa.

\section{Conclusion}

Since chronic subdural hematoma may cause KWNP it is important to have imaging done even in resource limited areas like Ghana in order not to do emergency exploratory burr holes on the wrong side of the brain.

\section{References}

1. Carrasco R, Pascual JM, Navas M, Martinez Florez P, Manzanares Soler R, et al. (2009) Kernohan-Woltman notch phenomenon caused by an acute subdural hematoma. J Clin Neurosci 16(12):1628-1631.

2. Kunii N, Morita A, Yoshikawa G, Kirino T (2005) Subdural hematoma associated with dural metastasis case report. Neurol Med Chir 45(10): 519-522.

3. Zhang CH, DeSouza RM, Kho JSB, Vundavalli S, Critchley G (2017) Kernohan Woltman notch phenomenon: a review article. British Journal of Neurosurgery 31(2): 159-166.

4. Kernohan JW, Woltman H W (1929) Incisura of the crus due to contralateral brain tumor. Arch Neurol Psychiatry 21: 274-278.

5. Tanaka A, Nakayama Y, Yoshinaga S (1997) Cerebral blood flow and intracranial pressure in chronic subdural hematomas. Surg Neurol 47(4): 346-351.
Creative Commons Attribution 4.0 International License

For possible submissions Click Here

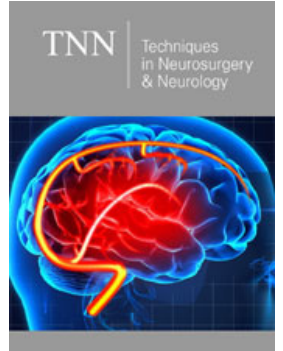

Techniques in Neurosurgery \& Neurology

\section{Benefits of Publishing with us}

- High-level peer review and editorial services

- Freely accessible online immediately upon publication

- Authors retain the copyright to their work

- Licensing it under a Creative Commons license

- Visibility through different online platforms 